\title{
"Age is associated with self-reported sleep bruxism, independently of tooth loss." A critical commentary
}

\author{
Ephraim Winocur
}

Received: 21 November 2011 /Revised: 21 November 2011 / Accepted: 22 November 2011 /Published online: 8 December 2011

(C) Springer-Verlag 2011

The article of Kato et al. [1] tries to give an answer to the paradoxical low prevalence of self-reported sleep bruxism (SB) in the elderly, independent of tooth loss.

According to the fourth edition of the Orofacial Pain Guidelines published by the American Academy of Orofacial Pain, bruxism is a diurnal or nocturnal parafunctional activity including clenching, bracing, gnashing, and grinding of the teeth [2]. The term bracing was defined by McNeill in the $>$ second edition of the Orofacial Pain Guidelines [3] as the following: "(syn, clenching) static, prolonged position of the mandible maintained by masticatory and tongue muscle activity, not necessarily involving contact of the teeth." The meaning is that it is possible that awake and sleep clenching (bracing) is partially performed without teeth contact.

SB has been considered for many years a dental occlusal disorder. It was believed that occlusal interferences cause a reflex-mediated activation of the elevator muscles through the stimulation of periodontal mechanoreceptors. Accordingly, the role of the bruxism activity is to eliminate occlusal interferences [4]. If this theory was true, bruxism must stop with dental loss. It is well known that sleep bruxism is also found in edentulous persons and in patients fully rehabilitated with implants [5]. Moreover, tooth contact has been found to occur for approximately $17.5 \mathrm{~min}$ over a 24-h period, and SB-related muscle activity lasted approximately $8 \mathrm{~min}$ over a complete sleep period [6]. Many studies tried to explain the possibility that a linear relation exists between SB, age, and tooth loss [1], but as far as I know, the

\section{E. Winocur $(\bowtie)$}

Department of Oral Rehabilitation, the Maurice and Gabriela

Goldschleger School of Dental Medicine, Tel Aviv University,

Tel Aviv 69978, Israel

e-mail: winocur@post.tau.ac.il association between self-reported SB and age, taking into account tooth loss, has never been investigated before the present study. Within the methodological limitations of the study, the conclusion of Kato et al. [1] was that self-reported $\mathrm{SB}$ is associated with age, independent of tooth loss. This result is in accord with the theory that SB is mainly centrally mediated, not peripherally [4]. The state of the art today is that despite the fact that the aetiology of sleep bruxism seems to be multifactorial (sleep homeostasis and arousal activity, oromotor excitability, psychological and personality traits, genetics, neurochemical activities, and oropharyngeal functions), there is no evidence of the role of occlusal or other morphological factors [7]. The finding of the current study that the prevalence of SB decreased with age was in accordance to similar studies performed all over the world, but for the first time this association was not confounded by tooth loss [1].

SB is classified by the International Classification of Sleep Disorders (II) as a sleep-related movement disorder [8]. The prevalence of most sleep movement disorders (e.g., periodic limb movement disorders and restless legs syndrome) increases with age, contrary to the decrease found in SB. As discussed by Kato et al. [1], the occurrence of SB and other sleep-related movements have been associated with endogenous sleep arousal activities such as microarousal and cyclic alternating patterns. Accordingly it was logical to anticipate that SB frequently would increase among elderly subjects since microarousal during sleep is known to occur often with increasing age. Nevertheless the contrary occurs. It was found that several neurochemicals (e.g., serotonin, dopamine, gamma aminobutyric acid, and noradrenaline) are involved in both the genesis of rhythmic jaw movements (e.g., bruxism) and the modulation of muscle tone during sleep [9]. With age physiological changes occur. A 
common impairment is neurotransmission. Investigations into the neurochemistry of the aging human demonstrated age-related changes in parameters of the serotonergic, cholinergic, and dopaminergic systems [10]. It may be possible that two opposite influences affect the prevalence of bruxism in the elderly. While arousal activity may provoke sleep bruxism, the neurotransmitter changes may attenuate it, leading to a net effect of lower prevalence.

Given the still numerous controversies, the association between SB and age has to be further investigated, but it is undisputable that the article of Kato et al. greatly contributes to the understanding of the SB aetiology.

\section{References}

1. Kato T, Velly AM, Nakane T, Masuda Y, Maki S (2012) Age is associated with self-reported sleep bruxism, independently of tooth loss. Sleep and Breathing (this issue)
2. De Leeuw R, Orofacial Pain (2008) Guidelines for assessment, diagnosis, and management, 4th edn. Quintessence, Chicago, p 263

3. McNeill C (1993) Temporomandibular disorders. Guidelines for assessment, diagnosis, and management, 2nd edn. Quintessence, Chicago, p 119

4. Lobbezoo F, Naeije M (2001) Bruxism is mainly regulated centrally, not peripherally. J Oral Rehabil 28:1085-1091

5. Galante JM (2010) Effects of bruxism on restorative implantassisted prosthesis treatments. In: Paesani D (ed) Bruxism theory and practice, chapter 223. Quintessence, London, pp 439-466

6. Lavigne GJ, Khoury S, Abe S, Yamaguchi T, Raphael K (2008) Bruxism physiology and pathology: an overview for clinicians. J Oral Rehabil 35:476-494

7. Kato T, Lavigne GJ (2010) Sleep bruxism: a sleep-related movement disorder sleep. Med Clin 5:9-35

8. American Academy of Sleep Medicine (AASM) (2005) The international classification of sleep disorders revised: diagnostic and coding manual (ICSD), 2nd edn. American Academy of Sleep Medicine, Westchester

9. Lavigne GJ, Kato T, Kolta A, Sessle B (2003) Neurobiological mechanisms involved in sleep bruxism. Crit Rev Oral Biol Med 14(1):30-46

10. Strong R (1998) Neurochemical changes in the aging human brain: implications for behavioral impairment and neurodegenerative disease. Geriatrics 53(Suppl 1):S9-S12 